Case report

\title{
Reducing HIV Mortality: A New Paradox for Practitioners Working in Countries with Socialized Health-care Systems
}

\author{
Pablo Goldschmidt \\ Received 31 July, 2008 Accepted 27 December, 2010 Published online 14 June, 2011
}

\begin{abstract}
The milestones marking substantial changes in the lives or in the survival of humans deserve to be remembered. It has been only 11 years since we experienced an event that not even the most optimistic amongst us would have predicted before 1997. Let us place the facts in time. At the beginning of the 80s, we faced the distressing reality that more than three quarters of all children, men and women found to have antibodies directed against a new infectious agent named human immune deficient virus (HIV) were bound to die. The mere reactivity of the serum of a human being against a virus characterized in 1983 (antibodies) handed an almost inevitable sentence of death. At that time the evolution of this viral infection was assessed by the quantification of a sub type of white cells, the auxiliary lymphocytes or CD4. This count was the principal evidence for most of the predictions on how a person might survive without degradation, and the value of such cells was the abacus used to forecast the time when an individual would develop irreversible blindness, to anticipate respiratory failure, and to predict the time before weakness would appear after devastating diarrhea, etc. We should recall that the CD4 cell count was even used as a predictor of the initiation of cognitive shrinkage, forecasting dementia as well as the signs that would take hold of personality as a consequence of infections or neoplastic transformations in the encephalitic mass.
\end{abstract}

Key words: AIDS, HIV, CD4 cells, Paradox, Efficient treatments, Survival, Generosity, Delusion, Power, Socialized Health Care System

\section{THE PREDICTIONS}

From the early 1980 s to 1997 , the task of providing assistance to HIV patients was perceived as futile. Health care establishments worked to alleviate pain amid a wall of resignation, receiving lab results in which the CD4 figures (surrogate markers) helped nurses, auxiliaries, students and doctors to hide their despair behind a protective fence built with objective cell numbers.

Before 1997 the sensation of powerlessness was gripping given that, aside from offering people with HIV the possibility to be included in research protocols to evaluate the primary or secondary prevention of opportunistic infections (pneumonia, encephalitis, retinitis, diarrhea), little could be done to limit the devastation triggered by the viral infection. The physician's work was limited to the description and assessment of technical events related to prognostication (CD4 count, levels of beta two micro-globulin or the intensity on the band of the protein P25 in the Western Blot test). Between 1985 and 1997 the surrogate marker results from the laboratories led to an attitude in the intimate forums of physicians that, as though they were fond of sport bets or prophecies, the circulating lymphocyte figures enabled clinicians to "predict" death in 8 months or less in some, or in less than 2 years in others cases.

At that time it caused constant distress trying to articulate the answer to the question of how, in the HIV infected patient's life, the decrease in CD4 cells creates a favorable environment for life threatening infections, for the growth of certain tumor masses and cancers of the immune system, adding physical and moral wasting signs and lethal risks to the pathology already triggered by this viral infection. The predictions were expressed in the context of anguish suffered by both patient and physician; the latter had to put into words the eventuality of the former's imminent and irreversible degradation.

When patients asked, "Now what should I do?" I recall frequently listening to colleagues-who were hostages of their own pessimism - provide answers loaded with syntactic constructions in the style of: "arrange your papers," or "make your will," or "seek advice with a lawyer," or "call your family and put your business in order."

Before 1997, the health workers treating people infected with HIV were overwhelmed with reservations about the appropriateness of prescribing and verifying the results of tests conceived to foretell physical degradation. In fact, once the auguries were established by this crop of scientific forecasts, no efficient therapeutics with long-term efficacy 
could be proposed. When treatments failed to protect the HIV-infected individual from pneumocistosis, cytomegalovirus, toxoplasmosis or mycobacterium infections, all that could be made to confront the immune collapse was to encourage the person, without any certainty, to fight empirically for life. At that time, in a humanistic framework of affection and understanding, it appeared even "helpful" to evoke in words the imminent arrival of the end of life.

From the position of the infected persons, the surrogate marker results were digested with resignation and fear, probably because the specific peculiarities of the transmission of this virus mixed guilt with suffering, and this enhanced the experience of anguish and hopeless expectations. Because confidentiality had to be guaranteed, many patients transformed suffering into inscrutability and silence. This was palpable when making the rounds of hospital beds where lonely human beings were coming to the end of their lives in conditions of affective and physical abandonment.

\section{TREATMENTS BEFORE 1997: STATE OF THE ART}

Approaching 1997, neither alternative medicine, nor macrobiotic diets, nor organic food, nor varied nutritive complements or supplements overloaded with vitamins and minerals, nor any kind of gym work or sport, nor sea water, nor thermal stations, nor essential oils, nor Western or Oriental massages, nor the consumption of oligoelements, nor antioxidants, nor the "reorganization and harmonization of the circulating energy of the body with that of the universe," nor yoga, nor tae-kwon-do, nor the good vibes of associative groups, nor witchcraft, nor transcendental meditation, nor the alkaloids of Cannabis sativa in any preparation or route of administration, nor the products obtained from Latin American cactus or fungi or exotic vegetables from any continent, nor the systemic or ex-vivo administration of cytokines, nor interferons, nor growth hormones, nor steroidal anabolic agents, nor heavy-metal associated organic molecules, nor preparations obtained following the formulae of Far Eastern or Western pharmacopoeias, nor passive immune therapeutics with hyper immune gamma globulin preparations, nor treatments with antigens of the virus, nor auto vaccines, nor derivatives of human placenta, nor the first antiretroviral strategies, exerted beneficial effects either on immune regeneration or on the quality of life of the infected persons, less even on the survival of the persons who had received the "declaration" of AIDS.

\section{EFFICACIOUS THERAPEUTICS}

Optimism rose in 1997 when it was proven worldwide that new antiviral strategies associating two or three synthetic antiviral compounds could reduce the mortality due to AIDS. In the United States the mortality decreased from $93 \%$ in 1981 to $6 \%$ at the end of 1997 , and the follow up of patients in Norway showed that the level of mortality between 1986 and 1990 (47\%) was dramatically decreased after 2006.

Several other trials confirmed these results worldwide. In Brazil it has been reported that the number of HIV infected people who had undergone antiretroviral treatment needing hospitalization and the number of deaths were significantly reduced after 1997 (the Brazilian population started to benefit from free antiretroviral therapeutics in 1996). However, the number of infected persons infected increased, indicating that access to free treatment requires consistent additional efforts to educate the population regarding the ways to avoid being contaminated by this virus.

In Barcelona, a constant increase in HIV-related mortality was observed until 1995, but the mortality curve showed and inflection and a significant decrease across almost the whole population from 1997. In this city, however, it should be noted that the treatments associated with antiretrovirals do not show an equivalent efficacy after stratifying the population according to the economic resources of the different groups of patients: the reduction in mortality was significantly lower in the layers of the population with the fewest resources and in persons who routinely intravenously inject psychoactive agents intravenously. Because in Spain the antiretroviral treatments are free of charge, the free treatment is an element that, although necessary to fight AIDS, does not sufficiently explain the discrepancy of the results obtained on mortality. Hence, to improve the quality of life and to prolong life expectancy, the intrinsic capacity of the antivirals to act on the specific molecular targets of the viral enzymes and the appropriate oral bioavailability of the pharmaceutical preparations should not be dissociated from other factors that determine clinical efficacy. Patients should actively adhere to the treatment and respect all the hourly indications ("compliance"), while health workers should first of all construct patient relationships which develop respect and affection with bonds of confidence, and secondly should educate infected and not-infected people alike, especially the population with a low level of education.

The encouraging results remove any doubt that since 1997 (when the therapeutic strategy known as post-exposition prophylaxis of the newborn baby was validated) antiretroviral treatments have limited the transmission of HIV from mothers to their babies due to a prominent reduction of the viral load in the blood of progenitors. Moreover, the first large studies conducted on pregnant women in 16 countries confirmed that the transmission of the virus from mothers 
could be limited if appropriate treatments were administered according to a specific dosage and schedule. Currently, the levels of transmission of HIV from mother to child range between 1 and $2 \%$ in highly industrialized countries, and the benefits of antiretrovirals have been demonstrated among toddlers with maternal milk as their only nutritional option.

\section{THE AWAKENING OF DELUSIONS}

The catastrophe triggered by AIDS became noticeable in Western countries in the early 1980 s, allowing certain individuals to undertake simultaneously the function of judge, jury, referee and contender. The newborn connoisseurs of AIDS overran the scientific and medical scene, and from this new arena they ruled the direction of work and qualified the relevance and value of investigations: the ex-nihilo structures played as unarguable commanders who decided on the pertinence of tasks to be conducted and the kind of information that deserved to be spread to society.

The rapid development of scientific leaders and state machines during the first year of the emergent epidemic can be viewed retrospectively as beneficial if this emanation was followed by decisions leading to the improvement of people's health. However, what was produced during those years reveals a picture in which AIDS only created-in countries with highly public concerns regarding health and socialized medicine-circles of power in which some flocked to the state network, rapidly enhancing their own careers and broadcasting their views as if they had received supreme revelations and dogmas on the infections and their evolution. This extensive occupancy of the chairs of AIDS expertise both basic research and medical treatment brought about quick and unexplained fame as well as the appointment of a legion of new medical professors. Those who in other geographical contexts would have been labeled "apparatchiks" made indisputable decisions regarding the appropriateness of who, why, where and how all the work related to AIDS research should be conducted.

During the first epidemic years, the investigation concerns were largely centered on imposed visions. Today, it deserves to be re-emphasized that the extent of human and financial resources allowed researchers to show, among others, the repeated issues imposing mycoplasma as the essential cofactor that determined the progress of a viral infection towards AIDS (today this processes are only limited by the association of antivirals).

Moreover, the dubious clinical relevance of administration of organic heavy metal salt complexes recalled and analyzed, as well as the treatment with cortisone to limit HIV evolution, or the benefits of palsmapheresis, or the implications of the supreme determination of the last identified lymphocyte or macrophage membrane associated protein or receptor and the overriding implications of other infectious agents different from HIV in the clinical degradation of people with HIV. It is amazing to realize that, at that time, many of those holding the presupposed "knowledge on AIDS" validated their work by their mere appearance in the mass media, and it happened frequently that many of their so-called truths were denied a posteriori.

As a paradigm of what was happening in science and medicine during the first years of the AIDS epidemic in France, generous donations for research were made to governmental and non-governmental institutions as the result of gifts flooding in from people with AIDS, most of whom were dead as a consequence of ineffective therapeutics. In addition, the cash influx to certain institutions increased from the mid-1980s and the mid-1990s thanks to a significant real estate patrimony that had been donated from people dying from AIDS or those in the advanced phase of infection who hoped that their gifts would contribute to the remedy of AIDS and help to finance the development of a vaccine. The citizens organized public performances, spectacles, concerts, fairs, and other highly-covered media events to raise funds for research to combat AIDS, and it was in the 1980s and 1990s that some French public notary waiting rooms kept pre-printed leaflets to be filled, dated and signed by a person so that his/her assets could be automatically transferred, posthumously, to organizations promoting AIDS research.

However, the results obtained by the recipients of these sums indicate that the expectations were not fulfilled and those of us who were in permanent contact with AIDS patients ask ourselves if the bequeathed assets have been effectively utilized. Let's remember that thanks to such generosity important sums were raised but were used to build facilities that did not fulfill the intentions of the legatee or pay for buildings where the AIDS authorities and their groups of followers might have expanded their ambitions. Our intention here is not to criticize the quality of the results, but the retrospective assessment of scientists' activities shows that the research was conceived, not only to approach the objectives desired by the legatees, but also to facilitate work in scientific areas which the researchers personally considered interesting or entertaining.

Simultaneously, in the public hospitals, the funds collected from generous donators and also from the national budget for public health were spent for a so-called "machine to produce papers". In public infrastructures a significant portion of the funding for work carried out in those years by the staff (whose salaries came from the taxpayers) was equivalent to what is offered for registration purposes by the private clinical research organizations to pharmaceutical 
corporations for phase II and phase III clinical trials. These CRO-like activities conducted by the medical staff increased the chances of civil servants or residents getting better positions, and at the bottom line, the health system built by the believers in socialized medicine was articulated as a public machine to feed resumes. Hence, this was also far from what the donors expected. However, because neither policy nor procedure taught at that time-when illness, suffering, donators, altruism, and generosity were mixed with good will- how to manage the funds, it seems senseless to accuse anyone a posteriori of inefficiency. Nevertheless, this peculiar situation reveals that the towers of fallacy and delusion which were built while struggling to reduce AIDS suffering should be burned into the future to avoid precipitated decisions and new virtual creatio ex-nihilo establishments. The birth and rapid growth of the HIV centers created an unfortunate picture that should be retained as a pattern to predefine what should never be done again.

\section{EFFICIENCY AND OPTIMISM AND THE EMER- GENCE OF A PARADOX}

AIDS generated a new unexpected ideological paradox that was inconceivable before 1997. In fact, results show that the HIV infection is no longer synonymous with a death sentence and persons who endure the consequences of bacterial, viral or parasitic infections in what was known as "declared AIDS" may recover their immune functionalities as well as their corporal mass and their aptitude to work, to love and to create. In countries with health systems offering free access to appropriate treatments, the lethal HIV infection has been transformed into a chronic controllable affliction. But none of these achievements were the result of appropriate public research policies, either of the product obtained from the avant-garde investigators who proved while working in public institutions to be eager to face the pertinent issues for the cure of AIDS, nor from acts of altruism!

If the life of people living with HIV improved, this was the result of the accurate, creative and systematic labors of chemists, biologists and clinicians who were pushing research forward from inside the pharmaceutical corporations, a statement that needs to be placed in the perspective of our primitive cultural perception of the original actors fighting against this infection. It appears today that not all was pristine that was generated by honest civil servants in their respective positions or that was the consequence of public research policies.

The HIV paradox deserves to be pointed out because, if since 1997 this viral infection is no longer synonymous with a death sentence, it is due almost solely to those who were considered to be unscrupulous "merchants of health".

The pharmaceutical industry was stigmatized and labeled as deprived of any kind of engagement in public health and only interested in medicines that produce quick and high turn over and profits by targeting pathologies affecting larger populations in rich countries. In congresses, meetings, scientific and extra scientific publications aired during the first years of the AIDS pandemic it was repeatedly stated that because AIDS affected a much smaller number of people than other pathologies and since in most of the HIV infected people income levels were low, the pharmaceutical corporations would be of little help. However, the enhancement of survival and quality of life and the reduction in physical and mental suffering was made possible by those from whom less was expected (while the big delusion came from those from whom much was expected!).

Finally, the purpose of presenting the concatenation of events that changed the life expectancy of HIV-infected people is neither to be trapped by indulgent and easy diatribes nor to put forth ideological pamphlet arguments that could have been drafted in the absence of proven evidence. Because the fight against AIDS shows a positive corollary, the assignment of blame to any particular establishment or individual may apparently have no impact. However, it appears that the presentation of questions for sustaining the future of good intentions and adroit decisions is salutary and that the dialectic analysis should be read as a prophylactic a posteriori auto critic, never pointing out faithlessness, inefficiency, prejudices, disorientation or misguidance. Therefore, the events that occurred between 1985 and 1997 should teach us how not to shape the appropriate strategies to face future emergent health crises without ruining good intentions, keeping in mind the crying need to push research policies forward because cancer is still killing massively, and politicians, media managers and fund raisers become agitated with the potential relevant returns of human distress.

In a regulated framework which respects universal ethical standards and free access to health care, a paradox was created by a "revolutionary" change operating in the management of HIV infection as a consequence of the speedy and efficient contribution from the private pharmaceutical sector. Because antiviral therapeutics have largely proven to reduce the fatal prognosis due to HIV, it still remains our urgent duty to share the success of the pharmacological strategies with our peers in countries with limited resources and with fellows living in industrialized countries, which, whether due to their socio-cultural, affective or educational situation require additional caring and dedicated efforts for education. 